\title{
The miR155HG/miR-185/ANXA2 loop contributes to glioblastoma growth and progression
}

\author{
Weining $\mathrm{Wu}^{1,2+}$, Tianfu Yu${ }^{1 \dagger}$, Youzhi $\mathrm{Wu}^{1,2+}$, Wei Tian², Junxia Zhang ${ }^{1}$ and Yingyi Wang ${ }^{1 *}$
}

\begin{abstract}
Background: Glioblastoma multiforme (GBM) is the most common and aggressive form of astrocytoma among adult brain tumors. Multiple studies have shown that long non-coding RNAs (IncRNAs) play important roles in acting as molecular sponge for competing with microRNAs (miRNAs) to regulate downstream molecules in tumor progression. We previously reported that miR155 host gene (miR155HG), an IncRNA, and its derivative miR-155 promote epithelial-to-mesenchymal transition in glioma. However, the other biological functions and mechanisms of miR155HG sponging miRNAs have been unknown. Considering ANXA2 has been generally accepted as oncogene overexpressed in a vast of cancers correlated with tumorigenesis, which might be the target molecule of miR155HG sponging miRNA via bioinformatics analysis. We designed this study to explore the interaction of miR155HG and ANXA2 to reveal the malignancy of them in GBM development.

Methods: The expression of miR155HG was analyzed in three independent databases and clinical GBM specimens. Bioinformatics analysis was performed to assess the potential tumor-related functions of miR155HG. The interaction of miR155HG and miR-185 and the inhibition of ANXA2 by miR-185 were analyzed by luciferase reporter experiments, and biological effects in GBM were explored by colony formation assays, EDU cell proliferation assays, flow cytometric analysis and intracranial GBM mouse model. Changes in protein expression were analyzed using western blot. We examined the regulatory mechanism of ANXA2 on miR155HG in GBM by gene expression profiling analysis, double immunofluorescence staining, chromatin immunoprecipitation and luciferase reporter assays.

Results: We found that miR155HG was upregulated in GBM tissues and cell lines. Bioinformatic analyses of three GBM databases showed that miR155HG expression levels were closely associated with genes involved in cell proliferation and apoptosis. Knocking down miR155HG suppressed GBM cell proliferation in vitro, induced a G1/S-phase cell cycle arrest, and increased apoptosis. We also found that miR155HG functions as a competing endogenous RNA for miR-185. Moreover, miR-185 directly targets and inhibits ANXA2, which exhibits oncogenic functions in GBM. We also found that ANXA2 promoted miR155HG expression via STAT3 phosphorylation.

Conclusion: Our results demonstrated that overexpressed miR155HG in GBM can sponge miR-185 to promote ANXA2 expression, and ANXA2 stimulates miR155HG level through phosphorylated STAT3 binding to the miR155HG promoter. We establish the miR155HG/miR185/ANXA2 loop as a mechanism that underlies the biological functions of miR155HG and ANXA2 in GBM and further suggest this loop may serve as a therapeutic target and/or prognostic biomarker for GBM.
\end{abstract}

Keywords: GBM, miR155HG, ANXA2, P-STAT3, ceRNA

\footnotetext{
* Correspondence: 373987405@qq.com

'Weining Wu, Tianfu Yu and Youzhi Wu these authors contributed equally to this work.

'Department of Neurosurgery, The First Affiliated Hospital of Nanjing Medical

University, Nanjing, Jiangsu 210029, China

Full list of author information is available at the end of the article
}

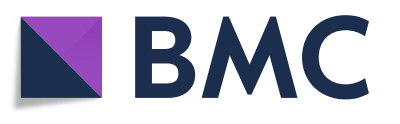

(c) The Author(s). 2019 Open Access This article is distributed under the terms of the Creative Commons Attribution 4.0 International License (http://creativecommons.org/licenses/by/4.0/), which permits unrestricted use, distribution, and reproduction in any medium, provided you give appropriate credit to the original author(s) and the source, provide a link to the Creative Commons license, and indicate if changes were made. The Creative Commons Public Domain Dedication waiver (http://creativecommons.org/publicdomain/zero/1.0/) applies to the data made available in this article, unless otherwise stated. 


\section{Background}

Glioblastoma multiforme (GBM) is the most aggressive form of astrocytoma and is characterized by rapid progression and poor prognosis [1]. Studies have indicated that GBM development is associated with disrupted gene regulatory networks. In addition to aberrant gene expression in GBM, several reports have demonstrated a role for non-coding RNAs (ncRNAs), which lack protein coding capability due the lack of an open reading frame, in GBM progression [2]. Among these ncRNAs, long ncRNAs (lncRNAs) are defined as RNAs > 200 nucleotides in length and have been demonstrated to participate in diverse cellular processes including epigenetics and transcriptional and posttranscriptional regulation [3]. At the posttranscriptional level, lncRNAs can act as competing endogenous RNAs (ceRNAs) or molecular sponge that function by sponging microRNAs (miRNAs), short endogenous ncRNAs approximately 18-25 nucleotides in length, resulting in inhibiting the effects of miRNAs on target mRNAs [4].

The lncRNA miR155HG is transcribed from a gene located on chromosome 21q21 and consists of three exons that span $1.5 \mathrm{~Kb}$. MiR155HG, also known as B-cell integration cluster, includes an imperfectly base-paired stem loop in exon 3 that is conserved across species and is a primary miRNA for miR-155 [5]. MiR155HG was initially thought to be involved in the human immune response. For example, Haasch et al. showed that transcriptional activation of miR155HG is an early and sustained $\mathrm{T}$ cell activation event [6]. Several oncological studies have shown that miR155HG is highly expressed in diffuse large and primary mediastinal B cell lymphomas [7]. MiR155HG can be induced by abnormal B-cell receptor in Hodgkin lymphoma [8], and miR-155 was also shown to be upregulated by mutant p53 and facilitate invasion of breast cancer cells [9]. Our group previously reported that the miR $155 \mathrm{HG} / \mathrm{miR}-155$ axis exhibits an oncogenic function in glioma by promoting epithelial-to-mesenchymal transition [10]. However, other biological mechanisms of miR155HG, such as potential ceRNA functions, and the upstream regulation of miR155HG in astrocytoma have remained unknown.

Annexin A2 (ANXA2) is a calcium-dependent phos pholipid-binding protein with demonstrated roles in stimulating fibrinolytic processes, degrading extracellular matrix, and promoting angiogenesis [11]. Recent reports have shown that ANXA2 is aberrantly expressed in a wide variety of tumors [12-15] and has been implicated in various processes of tumorigenesis, including cell invasion [16], proliferation [17] and neovascularization [18]. Previous studies reveal invasion function for ANXA2 in glioma and suggest its role as a potential diagnostic and prognostic marker for glioma [19, 20]. However, whether ANXA2 is involved in the oncogenic functions of miR155HG in glioma has not been determined.

Through bioinformatical analysis and experimental analyses, here we confirmed that miR155HG is overexpressed in GBM and acts as a ceRNA for the tumor suppressor miR-185 to upregulate ANXA2. We also showed that ANXA2 promotes GBM growth and miR155HG expression by activating STAT3. Our results demonstrate that miR155HG, miR-185 and ANXA2 form a signaling loop that promotes malignant phenotypes in GBM.

\section{Methods}

\section{Public human astrocytoma databases, GBM specimens} and cell lines

Three public human astrocytoma databases (TCGA, CGGA and Rembrandt) were described in our previous study [21]. A total of 24 GBM tissues and 15 pair-matched adjacent normal brain edematous tissues (collected postoperatively between April 2016 and February 2017) were collected from patients who underwent surgical removal of GBM tumors at the First Affiliated Hospital of Nanjing Medical University. Samples were frozen in liquid nitrogen immediately after isolation and stored at $-80^{\circ} \mathrm{C}$ to avoid RNA deterioration. Tumor tissues were collected after participants signed written informed consent. The study protocol was approved by The Institutional Review Board of the First Affiliated Hospital of Nanjing Medical University. GBM diagnoses were confirmed by two independent pathologists. Patients recruited into this study received no preoperative treatments.

The normal human astrocyte cell line NHA and the human GBM cell lines U87, U251, Ln229, T98, and A172 were purchased from the Chinese Academy of Sciences Cell Bank (Shanghai, China). A primary GBM cell line GP1 was extracted in December 2016 from the tumor of a patient with a temporal GBM; a second primary GBM cell line GP2 was extracted in January 2017 from the tumor of a patient with a frontal GBM. All cell lines were stored in liquid nitrogen before use. Cell culture was performed as described previously [22].

\section{Quantitative real-time PCR (qRT-PCR) and western blotting}

QRT-PCR and western blotting were performed as described previously [22]. The primer for miR155HG is F 5'-GAGTGCTGAAGGCTTGCTGT-3', R 5'-TTGA ACATCCCAGTGACCAG-3', for $\beta$-actin is $\mathrm{F} 5^{\prime}{ }^{\prime}$-TCAC CCACACTGTGCCCA-TCTACGA-3', R 5' - CAGCGG AACCGCTCATTGCCAATGG-3'. The antibodies for western blot analysis were: anti-ANXA2 (1:1000; Abcam, Cambridge, UK), anti-cell cycle-related proteins (cyclin E, cyclin D, CDK4, CDK6) (1:1000; Cell Signaling Technology, Danvers, MA, USA), anti-Bax (1:500; Santa 
Cruz Biotechnology, Dallas, TX, USA), anti-Bcl-2 (1:500; Santa Cruz Biotechnology), anti-caspase 3 (1:1000; Abcam) and anti- $\beta$-actin (1:1000, Cell Signaling Technology). Cells were treated with EGF Recombinant Human Protein Solution (Thermo Fisher Scientific, Waltham, MA, USA) or the SH-4-54 inhibitor of STAT3 phosphorylation (Selleck, Shanghai, China) according to the manufacturer's protocol.

\section{Oligonucleotides, plasmid and transfection}

To construct a plasmid expressing miR155HG, the full-length human miR155HG sequence or a mutated miR155HG sequence for miR-185-5p (NCBI Reference Sequence: NR_001458.3) was synthesized and inserted into the pCDNA3.1 vector to generate pCDNA3.1-miR155HG WT or MUT, respectively (Genechem, Shanghai, China). The hsa-miR-185-5p mimic, hsa-miR-185-5p inhibitor, and hsa-miR-scramble were chemically synthesized (Ribobio, Guangzhou, China). The sequence of ANXA2 and miR155HG siRNAs were as follows: si-miR155HG: sense, 5'-CUGGGAUGUU-CAACCUUAATT-3', antisense, 5'-U UAAGGUUGAACAUCCCAGTT-3'; scramble: sense, $5^{\prime}$-U UCUCCGAACGUGUCACGUTT-3', antisense, 5'-ACGU GACACGUU-CGGAGAATT-3'. ANXA2 siRNAs were chemically synthesized (Invitrogen, Shanghai, China). ANXA2 siRNA 1 target sequence is $5^{\prime}$-CUGGGAAGAAGG CUUC-CUUTT-3', ANXA2 siRNA 2 target sequence is 5'-AAGGAAGCCUUCUUCCC-AGTT-3', ANXA2 siRNA 3 target sequence is $5^{\prime}$ - GGGAAGAAGGCUUCCU-UCAT T-3'. Cells were transfected with oligonucleotides or plasmid using Lipofectamine 2000 Reagent (Invitrogen, Carlsbad, CA, USA) following the manufacturer's instructions.

\section{Lentiviral packaging and stable cell lines}

Lentiviruses carrying shRNA-miR155HG or shRNAANXA2 and the negative control lentivirus (sh-miR 155HG sequence is 5'-CUGGGAUGUUCAACCUUAA TT-3'; sh-ANXA2 sequence is 5' - CGGGATGCTTTG AACATTGAA - $3^{\prime}$; sh-NC sequence is $5^{\prime}$-UUCUCCG AACGUGUCACGUTT-3') were assembled in the human embryonic kidney cell line $293 \mathrm{~T}$, and the viruses were collected according to the manufacturer's manual (Genechem). Stably transfected cell lines were established by infecting U87 cells with lentiviruses using a lentiviral packaging kit purchased from Genechem, followed by puromycin selection.

\section{Chromatin immunoprecipitation (ChIP)}

ChIP assays were performed as previously described [22]. The EZ-magna ChIP kit (Millipore, Bedford, MA, USA) was used according to the manufacturer's protocol. Crosslinked chromatin was sonicated into DNA fragments in the range of 200-1000 bp and immunoprecipitated using rabbit anti-p-STAT3 antibodies (Abcam). Negative control samples were prepared using control rabbit anti-IgG antibody (Abcam), and rabbit anti-RNA Polymerase II antibody (Abcam) was used for positive control. After immunoprecipitation, the beads were washed sequentially with low-salt buffer, high-salt buffer, $\mathrm{LiCl}$ buffer, and TE buffer each for $5 \mathrm{~min}$ at $4{ }^{\circ} \mathrm{C}$. The immunoprecipitated DNA was then eluted by incubation in $100 \mu \mathrm{l}$ of elution buffer $\left(0.1 \mathrm{M} \mathrm{NaHCO}_{3}\right.$ and $1 \%$ SDS $)$ containing $10 \mu \mathrm{g}$ proteinase $\mathrm{K}$ (Millipore) at $62{ }^{\circ} \mathrm{C}$ for 2 $\mathrm{h}$ with rotation. The eluted DNA was purified using the columns and buffers in the kit and then re-dissolved in $50 \mu \mathrm{l}$ of PCR-grade water. Immunoprecipitated chromatin was analyzed by $\mathrm{qPCR}$ using primers targeting the phosphorylated (p)-STAT3 (p-STAT3) binding regions in the human miR155HG promoter. The primer sequences used for ChIP-qPCR are binding region 1 ( -1982 to 1972): F 5'-GAGACATCATTATTGTCATT-3', R 5'-TA GGAGTCAAATACACCTG-3'; binding region 2 (1548 to -1411 ): $\mathrm{F}$ 5'-ATGGGAAATTCAGAAAGGC -3', R 5' -TGATCATATGAGGGAGGAGC-3'; and binding region 3 (-275 to -116$)$ : $F$ 5'-TTAAGAACA AAGGTTGGAGC-3', R 5'-TGTGACTCATAACCGAC CAG-3'. PCR conditions were set according to the instructions provided in the SYBR Green Kit (Roche Applied Science, Upper Bavaria, Germany). Results were analyzed by agarose gel electrophoresis.

\section{RNA immunoprecipitation (RIP)}

RIP assays were performed using U87 cell extracts with the EZ-Magna RIP RNA-Binding Protein Immunoprecipitation Kit (Millipore, Burlington, MA, USA) according to the manufacturer's instructions. U87 cells were rinsed with cold PBS and fixed with $1 \%$ formaldehyde for $10 \mathrm{~min}$. After centrifugation, cell pellets were collected and resuspended in NP-40 lysis buffer (Thermo Fisher Scientific, Waltham, MA, USA) supplemented with $1 \mathrm{mM}$ PMSF, $1 \mathrm{mM}$ DTT, 1\% Protease Inhibitor Cocktail (Sigma-Aldrich, St. Louis, MO, USA) and 200 U/ml RNase Inhibitor (Life Technologies, Carlsbad, CA, USA). Lysates were subjected to high-speed centrifugation, and then $100 \mu \mathrm{l}$ of the supernatant was incubated with RIP buffer containing magnetic beads conjugated with human anti-Ago2 antibody (Cell Signaling Technology). Mouse IgG (Cell Signaling Technology) was used as a negative control, while SNRNP70 (Cell Signaling Technology) was used as a positive control. Co-precipitated RNAs were detected by reverse transcription PCR. Total RNAs (input control), IgG and SNRNP70 were assayed simultaneously to evaluate the efficiency of Ago2-specific RNAs.

\section{Colony formation assays}

Cells $\left(4 \times 10^{2}\right)$ were seeded into cell culture dishes and cultured for $15 \mathrm{~d}$. Cell colonies were fixed with $4 \%$ paraformaldehyde for $20 \mathrm{~min}$ and stained with $0.2 \%$ crystal violet. Images were captured and colonies (diameter $>$ $0.5 \mathrm{~mm}$ ) were counted using Image J software (National 
Institutes of Health, Arlington, VA, USA). All assays were repeated at least three times.

\section{EDU cell proliferation assays}

EDU cell proliferation assays were conducted with the Molecular Probes EdU-Alexa imaging detection kit (Life Technologies). Cells treated for $48 \mathrm{~h}$ were incubated with $10 \mu \mathrm{M}$ EdU for $2 \mathrm{~h}$, fixed with $4 \%$ paraformaldehyde, permeabilized with $1 \%$ Triton X-100, and stained with the Alexa-Fluor 594 reaction cocktail for EdU and Hoechst 33342 (nuclei). Images were obtained using a fluorescence microscope (Olympus, Japan). All assays were repeated at least three times.

\section{Flow cytometric analysis}

Transfected GBM cells in logarithmic growth were collected and processed with the Cell Cycle Staining Kit (Multi Sciences, Hangzhou, China) for cell cycle analysis. After washing with PBS, cells were fixed with 70\% ice-cold ethanol, incubated with Cell Cycle Staining Kit for $30 \mathrm{~min}$ in the dark, and analyzed by flow cytometry. In other experiments, treated cells in logarithmic growth were harvested and stained with the Annexin V-FITC Apoptosis Detection Kit (Multi Sciences). After washing with PBS and incubating with Annexin V/propidium iodide for $30 \mathrm{~min}$ in the dark, cells were analyzed by flow cytometry.

\section{Immunohistochemistry (IHC)}

Fresh intracranial tumor tissues from nude mice were fixed with $4 \%$ paraformaldehyde and then embedded in paraffin. Sections were incubated at $4{ }^{\circ} \mathrm{C}$ overnight with primary antibodies against ANXA2 (1:1000; Abcam) and p-STAT3 (1:500; Abcam). Sections were then incubated with secondary antibody (1:1000; Santa Cruz) for $2 \mathrm{~h}$ at room temperature and stained with diaminobenzidine until brown granules appeared.

\section{Fluorescence in situ hybridization (FISH)}

RNA FISH was performed as described previously [22]. MiR155HG-Bio probe was synthesized from GoodBio (Wuhan, China); the sequence is 5'-CCTCCCACG GCAGCAATTTGTTCCA-3'. Frozen sections of fresh tissues were fixed with $4 \%$ formaldehyde for $10 \mathrm{~min}$, washed with PBS, and then digested with Proteinase $\mathrm{K}$ for $5 \mathrm{~min}$. After eliminating auto-fluorescence and blocking endogenous biotin, the sections were hybridized with probes overnight. Sections were then washed with pre-warmed $2 \times \mathrm{SSC}$ at $37^{\circ} \mathrm{C}$ for $10 \mathrm{~min}, 1 \times \mathrm{SSC}$ at $37^{\circ} \mathrm{C}$ for $10 \mathrm{~min}$, and $0.5 \times \mathrm{SSC}$ for $10 \mathrm{~min}$. Tissue sections were then blocked with bovine serum albumin for 30 min at room temperature, followed by staining with 488-avidin (1:400) at room temperature for $50 \mathrm{~min}$. Stained sections were washed with PBS for 5 min four times. Finally, tissue sections were mounted with a medium containing DAPI for $8 \mathrm{~min}$ in the dark and images were obtained with fluorescence microscope (Nikon, Japan).

\section{Dual-luciferase reporter assay}

The ANXA2 3'-untranslated region (UTR) and the full miR155HG sequence containing miR-185-5p seed matching sites were amplified from human cDNA via PCR and cloned into the $3^{\prime}$ end of the pGL3-basic luciferase vector (Genechem). Mutated versions of each construct were generated by mutating the miR-185-5p seed site sequences (pGL3-wt or mut). The miR155HG promoter region sequence (2000 bp to $1000 \mathrm{bp}$ upstream of transcription starting point) were also amplified and cloned into the $5^{\prime}$ end of the pGL3-basic luciferase vector. Mutated version was generated by deleting binding region sequences of p-STAT3 (wt or mut-pGL3). U87 cells seeded into 96-well plates were co-transfected with wt or mut report gene, the pRL-TK control (Promega, Madison, WI, USA) and miR-185-5p mimic or miRNA NC using Lipofectamine 2000 (Invitrogen). The wt or mut-pGL3 and the pRL-TK control were co-transfected into the cells, then treated with cell culture with or without SH-4-54 inhibitor of STAT3 phosphorylation. At 48 $\mathrm{h}$ after transfection, luciferase activity was determined using the Dual Luciferase Reporter Assay System (Promega, WI, USA) according to the manufacturer's protocol. The relative luciferase activity was normalized to Renilla luciferase activity. All assays were performed in triplicate.

\section{Intracranial GBM mouse model}

The animal experiments were approved by the Animal Management Rule of the Chinese Ministry of Health (document 55, 2001) and were performed conforming to the approved guidelines and experimental protocols of Nanjing Medical University. U87 cells $\left(1 \times 10^{6}\right)$ stably expressing MCS-firefly luciferase for bioluminescence imaging were transfected with lentivirus expressing control shRNA, shRNA-ANXA2 or shRNA-miR155HG and then were intracranially injected into the frontal lobe of nude mice to generate GBM ( $n=10$ mice per group). Tumor volumes were measured by luciferase using a bioluminescence imaging system (Caliper IVIS Spectrum, PerkinElmer, Waltham, MA, USA) on days 1, 11, and 21 after implantation. The integrated flux of photons (photons/s) within each region was determined by the Living Images software package (Caliper Life Sciences). Mice were sacrificed when they were in deep coma. Brains were extracted, fixed in $10 \%$ formalin and then embedded in paraffin for IHC or frozen at $-80^{\circ} \mathrm{C}$ for western blotting or FISH. 


\section{Statistical analysis}

Data are presented as the mean \pm standard deviation (SD). Statistical analyses were performed using the Student $t$ test to evaluate the significance of differences between groups, one-way ANOVA (Tukey's post hoc) was used to determine the difference among at least three groups using SPSS v19.0 for Windows. (SPSS, IL, USA). Pearson's correlations analysis and heat map microarray analysis were performed using Multiple Array Viewer 4.9 software (MEV). Kaplan-Meier survival analysis was performed using GraphPad 5.0 software. $P<0.05$ indicates a significant difference.

\section{Results}

\section{MiR155HG is overexpressed in GBM and miR155HG-} related genes are enriched in cancer-associated processes To explore miR155HG expression in human astrocytoma tissues, we examined three public human astrocytoma databases (TCGA, CGGA and Rembrandt) and found overexpression of miR155HG in GBM (Fig. 1a). We also found that miR155HG expression was elevated in 24 GBM specimens compared with adjacent normal brain tissue from patients histologically diagnosed with GBM, and miR155HG was generally overexpressed in the GBM cells compared with NHA cells from normal brain tissue using fluorescent qPCR (Fig. 1b). FISH analysis revealed that miR155HG was primarily concentrated in the cytoplasm of GBM cells (Fig. 1c).

MEV software was used to perform a Pearson's correlation analysis to identify genes associated with miR155HG expression in the TCGA, CGGA and Rembrandt GBM databases. The results identified 1037 up-regulated genes and 873 down-regulated genes from the overlap of the three databases, and these genes were examined using the DAVID Web tool (http://david.abcc. ncifcrf.gov/home.jsp) for Gene Oncology (GO) and KEGG Pathway enrichment analyses. GO analysis showed that the upregulated genes were primarily enriched in tumor progression processes, such as regulating apoptosis and cell proliferation. Moreover, KEGG pathway analysis indicated that the upregulated genes were closely associated with pathways activated in cancers, such as the PI3K-Akt signaling pathway, a prominent pathway in tumorigenesis and cancer progression (Fig. 1d-e). Furthermore, gene set enrichment analysis (GSEA) (http://www.broadinstitute.org/gsea/index.jsp) was used to examine genes that were expressed in TCGA GBM samples from patients with high miR155HG expression and those with low miR155HG expression. Genes expressed in patients with high miR155HG expression were primarily associated with reduced apoptosis and cell death, while genes with negative expression were primarily associated with cell cycle progression and cell cycle phase transition (Fig. 1f-g).
Together these results suggested that miR155HG may be involved in the malignant phenotypes of GBM.

To evaluate the role of miR155HG in promoting malignant phenotypes of GBM, we generated an intracranial GBM mouse model by injecting U87 cells infected with lentivirus expressing control shRNA or shRNA-miR155HG. The tumor volumes in the group of mice treated with shRNA-miR155HG were smaller than those in the control at 11 and 21 days after implantation. Furthermore, mice treated with shRNA-miR155HG showed better survival than controls (Additional file 1: Figure S1A and B). These results indicated that miR155HG acted as oncogene in promoting GBM growth.

\section{MiR155HG sponges and downregulates miR-185-5p}

Previous studies have shown that lncRNAs can act as a sponge for miRNAs, therefore we speculated that miR155HG may exhibit sponge activity. RNA hybrid bioinformatics tools showed that miR155HG contains a putative binding site for miR-185-5p as tumor suppressor in a wide range of tumors [23-26] (Fig. 2a). We found that miR-185-5p levels were lower in GBM tissues than in normal brain tissue, and that miR-185-5p was significantly negatively correlated with miR $155 \mathrm{HG}$ in the same GBM samples (Fig. 2b; $r=-0.5970, p=0.0021$ ).

RNA-induced silencing complex (RISC) is an essential factor in the biological effect of miRNAs, and Ago2 is an elementary catalytic constituent of RISC that is involved in RNA cleavage [27]. To explore the possible interactions between miR155HG and miR-185-5p, RIP was performed in U87 cells. SNRNP70 protein, which interacts with U1 spliceosomal RNA [28], was used as a positive control. MiR155HG was predominantly enriched in beads containing anti-Ago2 antibody compared with those harboring control IgG (Fig. 2c). This result suggests that miR155HG is capable of sponging miRNA as ceRNA.

Then we constructed a luciferase reporter plasmid containing the putative miR-185-5p binding site from miR155HG, as well as a mutant construct in which the binding site was mutated (Fig. 2a). Co-transfecting miR-185-5p mimic decreased the relative luciferase activity in U87 cells transfected with wild-type plasmid, but had no impact on the mutant construct (Fig. 2d), which suggests that miR155HG directly binds miR-185-5p.

\section{ANXA2 is the target molecule of miR-185-5p}

Previous studies demonstrated a potential role for ANXA2 in glioma [19]. Bioinformatics analytical tools TargetScan and miRNAWalk 2.0 showed that the 3'-UTR of ANXA2 mRNA contained a seed sequence of miR-185-5p (Fig. 2e). To determine whether ANXA2 may be involved in the miR155HG-miR-185-5p axis in GBM, we first examined the expression levels of ANXA2 in frozen GBM tissue samples by western blot. We 


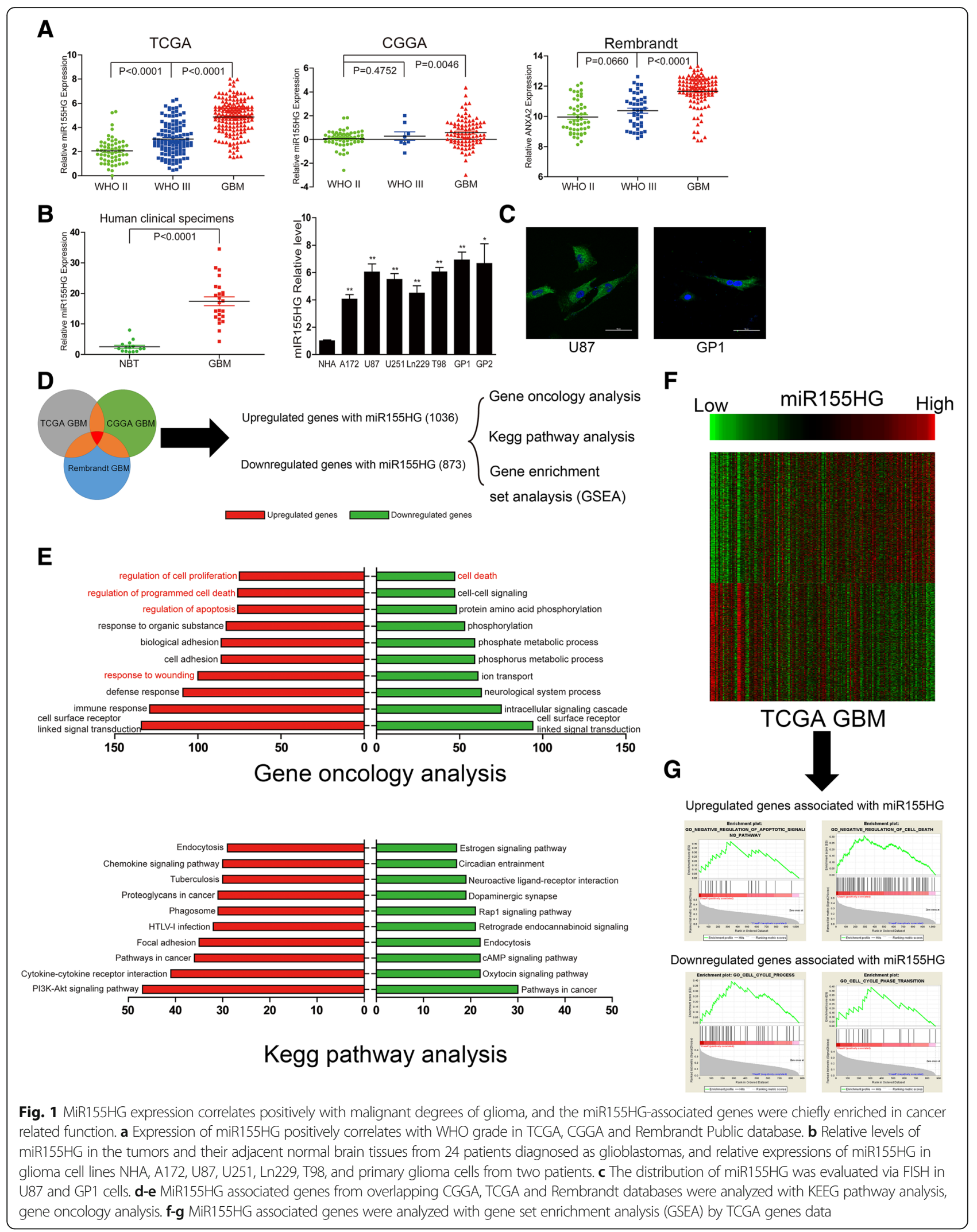




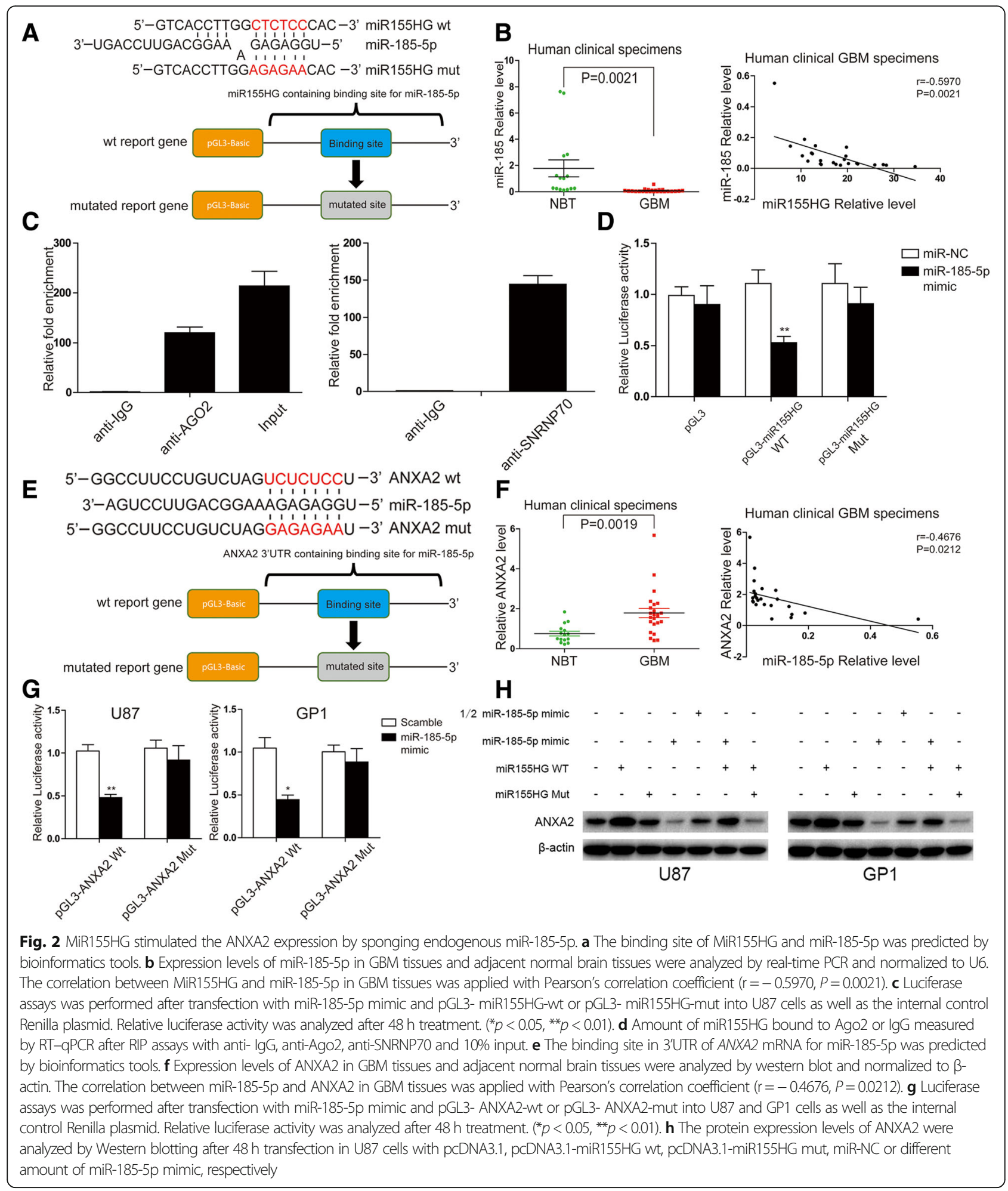

found that ANXA2 was highly expressed in GBM tissue but not in normal brain tissue (Fig. $2 \mathrm{f}$ and Additional file 1: Figure S1C). We next examined the correlation between miR-185-5p and ANXA2 in GBM tissue and found that miR-185-5p negatively correlated with ANXA2 $(r=$
0. $-4676, P=0.0212$; Fig. 2f). We generated luciferase constructs containing either the wild-type (WT) 3 '-UTR of ANXA2 mRNA or a mutated (MUT) sequence in which the miR-185-5p seed sequences were mutated. Luciferase assays showed that expression of miR-185-5p decreased 
the luciferase activity of the WT reporter but not the activity of the MUT reporter in U87 and GP1 cells (Fig. 2g).

We speculated that ANXA2 levels in GBM cells may be regulated by miR-185-5p and affected by its interaction with miR155HG. Indeed, transfection of a miR155HG expression vector increased ANXA2 levels in U87 and GP1 cells; however, the vector expressing miR155HG with mutated binding sites for miR-185-5p had no effect on ANXA2 levels. In addition, miR155HG-mediated elevation of ANXA2 was blocked by co-transfection with miR-185-5p mimic in a dose-dependent manner (Fig. 2h). Furthermore, inhibiting miR155HG by siRNA downregulated ANXA2 levels in U87 and GP1 cells, which could be reversed by treatment with miR-185-5p inhibitor (Additional file 1: Figure S1D). Together these results demonstrated that miR155HG may promote ANXA2 expression by modulating the capacity of miR-185-5p to bind the 3 '-UTR of ANXA2 mRNA.

ANXA2 enhances the malignant phenotypes of GBM cells As ANXA2 was the downstream molecule positively modulated by miR155HG via the ceRNA mechanism, we needed to investigate the function of ANXA2 to explain the oncological role of miR155HG in GBM. Bioinformatics analysis showed that ANXA2 expression was mostly expressed in GBM samples from TCGA, CGGA and Rembrandt database (Additional file 2: Figure S2A). GO analysis showed ANXA2 was closely associated with genes involved in cell apoptosis and proliferation (Additional file 2: Figure S2B).

We next performed a series of experiments to evaluate the possible oncogenic function of ANXA2 in GBM. Colony formation and EDU assays were performed in cells transfected with ANXA2 siRNA to evaluate the effect of ANXA2 on proliferation. The effect of ANXA2 siRNA was shown in Additional file 3: Figure S3B. A significant reduction of proliferation was observed in U87 and GP1 cells transfected with ANXA2 siRNA compared with controls (Fig. 3a-b). Flow cytometry revealed that knocking down ANXA2 induced a G1/S arrest and decreased the percentage of cells in S phase (Fig. 3c). Flow cytometry also showed that knocking down ANXA2 remarkably increased apoptosis rates in GBM cells (Fig. 3d). Western blot results of proliferation- and apoptosis-associated proteins were consistent with the above results (Fig. 3e).

To examine the oncogenic ability of ANXA2 in GBM in vivo, nude mouse tumorigenicity assays were performed using a U87 xenograft model. U87 cells infected with fluorescent lentiviruses expressing sh-ANXA2 or controls were injected into nude mouse brains. The effect of ANXA2 knockdown in cells and GBM tissue was shown in Additional file 1: Figure S1E. In vivo imaging of the nude mice at 1,11 and $21 \mathrm{~d}$ after implantation revealed that tumor growth was significantly inhibited at $21 \mathrm{~d}$ in the group with decreased ANXA2 expression (Fig. 3f). Mice injected with cells expressing sh-ANXA2 also showed better survival than control mice (Fig. 3g). IHC of tumor sections from the sh-ANXA2 and control groups confirmed decreased ANXA2 levels lead to inhibition of tumor growth in vivo (Fig. 3h). Taken together, our in vitro and in vivo results demonstrate that ANXA2 exhibits oncogenic functions in GBM cells and enhances the malignant phenotypes of tumors.

\section{MiR155HG and miR-185-5p participate in GBM growth by regulating the proliferation and apoptosis of GBM cells} As ANXA2 plays a crucial tumor-promoting role in GBM and since ANXA2 levels are modulated by miR155HG and miR-185-5p, we hypothesized that miR155HG and miR-185-5p could interfere with the proliferation and apoptosis of GBM cells. Downregulating miR155HG levels in U87 and GP1 cells by siRNA reduced proliferation, blocked cell cycle progression, and stimulated apoptosis in GBM cells, and these effects were reversed by miR-185-5p inhibitor (Fig. 4a-d). Western blot analysis showed altered levels of proteins associated with proliferation and apoptosis, which was consistent with the above results (Fig. 4e). These results indicate that the biological role of miR155HG and miR-185-5p in GBM cells was due to regulating proteins associated with proliferation and apoptosis, and this may be partially by regulating ANXA2 expression.

\section{ANXA2 affected miR155HG expression in GBM cells via p- STAT3 levels}

Our results show that miR155HG can interfere with ANXA2 expression by sponging miR-185-5p in GBM cells. Previous studies show that ANXA2 can act with AKT, STAT3 to promote downstream oncogenes [29, 30], we speculate if ANXA2 can contribute to miR155HG aberrantly overexpression in GBM cells in this way. Pearson's correlation analysis showed that ANXA2 was significantly positively correlated with miR155HG in the TCGA, CGGA and Rembrandt GBM databases (Fig. 5a). We also found a positive correlation between ANXA2 and miR155HG in WHO II and WHO III astrocytoma patients in these independent public databases (Additional file 2: Figure S2C). Next, one representative GBM tissue exacted from a GBM patient during surgery was examined by FISH double staining and the results showed that ANXA2 and miR155HG were both significantly expressed in dense tumor tissues, but not in loose normal brain tissue (Fig. 5b). Then double-staining was performed to investigate to show that inhibiting ANXA2 caused a miR155HG downregulation in the brain of nude mice from Fig. $3 g$ and interfered with tumor growth (Fig. 5c). We thus concluded that 


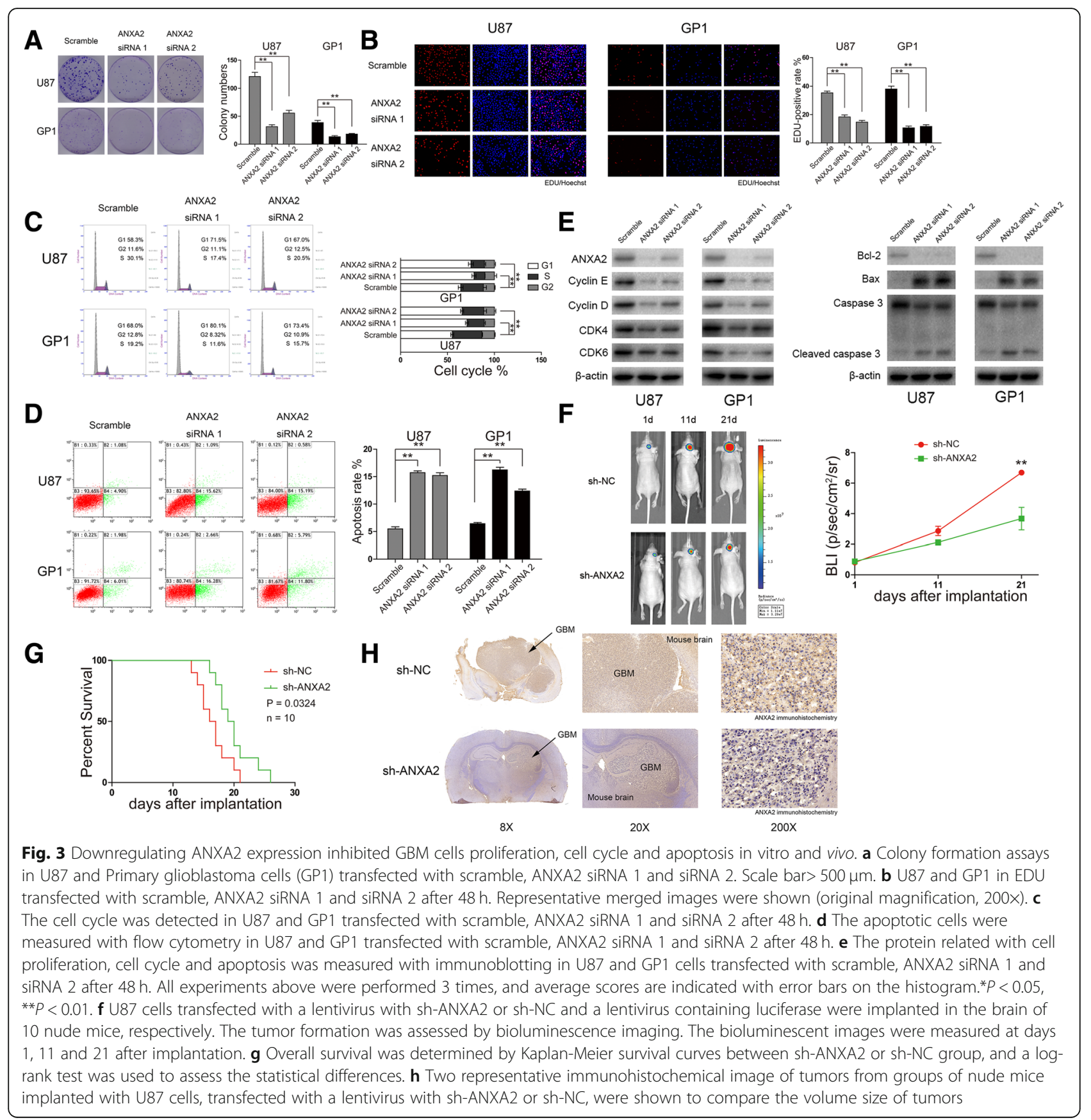

ANXA2 expression positively correlated with miR155HG levels in GBM.

Previous studies showed that ANXA2 modulates STAT3 phosphorylation (p-STAT3) levels to stimulate proliferation, angiogenesis, metastasis and epithelial to mesenchymal transition in breast cancer cells [30-32]. We found higher levels of p-STAT3 in GBM cells and tissues compared with normal brain cell and tissue(Additional file 3: Figure S3A), with distribution in nuclei in GBM cells (Fig. 5d). We thus wondered if ANXA2-mediated induction of miR155HG involved p-STAT3. We first found that decreasing ANXA2 resulted in reduced levels of p-STAT3 without impacting STAT3 levels in GBM cells (Additional file 3: Figure S3B). Inhibiting miR155HG also resulted in decreased ANXA2 and p-STAT3 in vivo (Additional file 3: Figure S3E). Knocking down ANXA2 resulted in reduced miR155HG expression, but this effect was not observed in GBM cells treated with a STAT3 phosphorylation inhibitor (Fig. 5e). Moreover, induction of STAT3 phosphorylation by Epidermal Growth Factor (EGF) [33, 34] could rescue the inhibitory effect of ANXA2 depletion on miR155HG expression (Fig. 5f and Additional file 3: Figure S3C). These results suggested that 


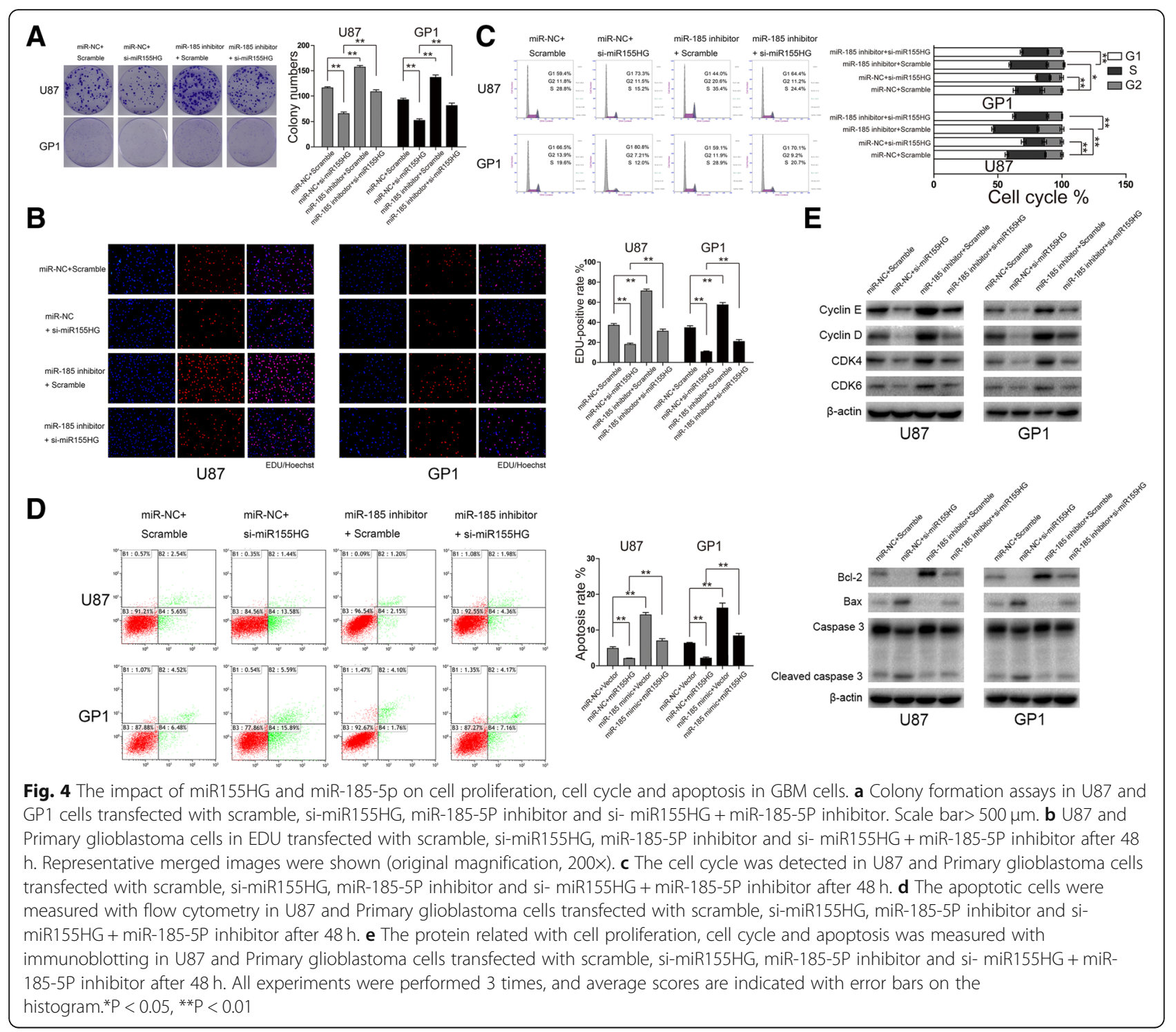

p-STAT3 might directly promote miR155HG expression in GBM.

Bioinformatics tools identified three putative binding regions for p-STAT3 in the miR155HG promoter. ChIP assays confirmed that $\mathrm{p}-\mathrm{STAT} 3$ could bind putative binding region 2 ( $-1548 \mathrm{bp}$ to $-1411 \mathrm{bp}$ ) but not binding region 1 ( -275 bp to $-161 \mathrm{bp}$ ) and binding region 3 (-1982 bp to -1972 bp) in the miR155HG promoter (Fig. 5g). Two luciferase reporter plasmids containing either the entire miR155HG promoter region $2000 \mathrm{bp}$ to $1000 \mathrm{bp}$ upstream of the transcription start site (wt-pGL3) or the promoter deleted for binding region 2 (mut-pGL3) were transfected into GBM cells along with or without STAT3 phosphorylation inhibitor. While inhibiting STAT3 phosphorylation resulted in downregulation of luciferase activity driven by the wild-type promoter, the p-STAT3-driven luciferase activity on the mutated reporter remained unchanged (Additional file 3: Figure S3D).
Together these results indicated that the activated transcription factor p-STAT3 plays a key role in ANXA2-driven miR155HG expression and promotes GBM cell growth, through the DNA binding activity of the p-STAT3 transcription factor.

\section{Discussion}

We previously revealed that miR-155, which is derived from miR155HG, functions in epithelial-mesenchymal transition in glioma [10]. In this report, we further showed that the lncRNA miR155HG is highly expressed in GBM, where it acts as a ceRNA to sponge miR185-5p, thus promoting miR155HG downstream molecules level such as ANXA2. Our study clarifies the mechanism by which miR155HG positively regulates AXNA2 to sustain the malignant phenotypes of astrocytoma, particularly GBM. 


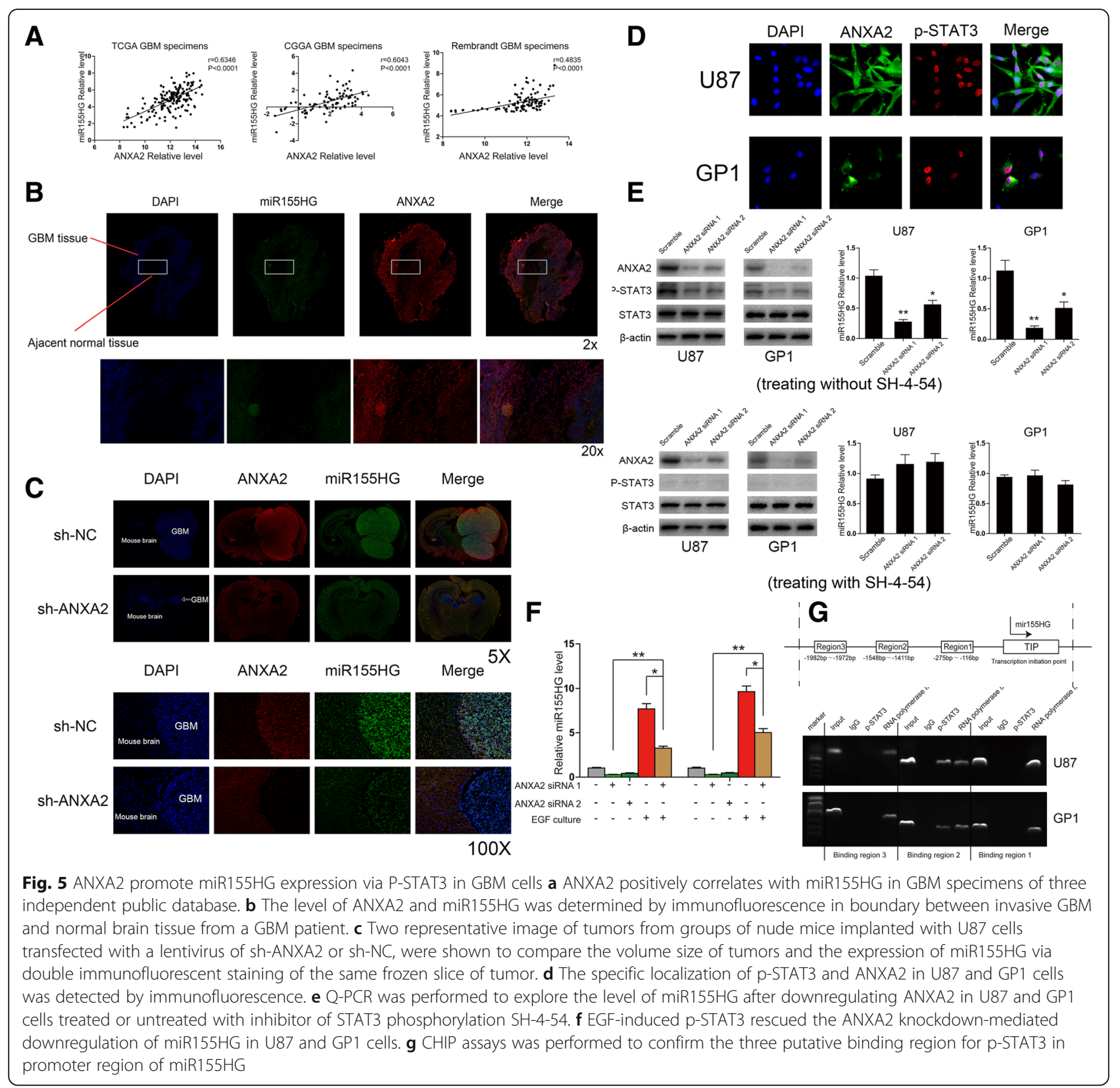

Through bioinformatics analyses, we found that miR155HG was closely associated with the proliferative activity and apoptosis resistance of GBM in three independent GBM gene expression arrays. Knocking down miR155HG in GBM cells resulted in cell cycle arrest, decreased cell growth, and apoptosis. As lncRNAs have been proven to function as miRNA sponges [35], we hypothesized that miR155HG might also regulate gene expression by competing for shared miRNA response elements in GBM. Several studies have shown that miR-185 is involved in suppressing non-small cell lung cancer [36], gastric cancer [37], hepatocellular carcinoma [38] and prostate cancer [39] and is downregulated in glioma associated with inhibiting glioma cell invasion [40]. Another report showed that the lncRNA Linc00176 regulates the cell cycle by sponging miR-185 in hepatocellular carcinoma [41]. Here, we confirmed that lncRNA miR155HG binds to miR-185 to impact proliferation, cell cycle progression and apoptosis in GBM cell lines.

ANXA2, a $36-\mathrm{kDa}$ protein that belongs to the family of calcium-dependent phospholipid binding proteins [11], is a DNA-binding protein that modulates DNA synthesis. Several studies showed that ANXA is involved in cell proliferation and cell cycle progression [42-45] in a variety of cancer cell types, such as breast cancer [18], hepatocellular carcinoma [46], colorectal cancer [47] and 


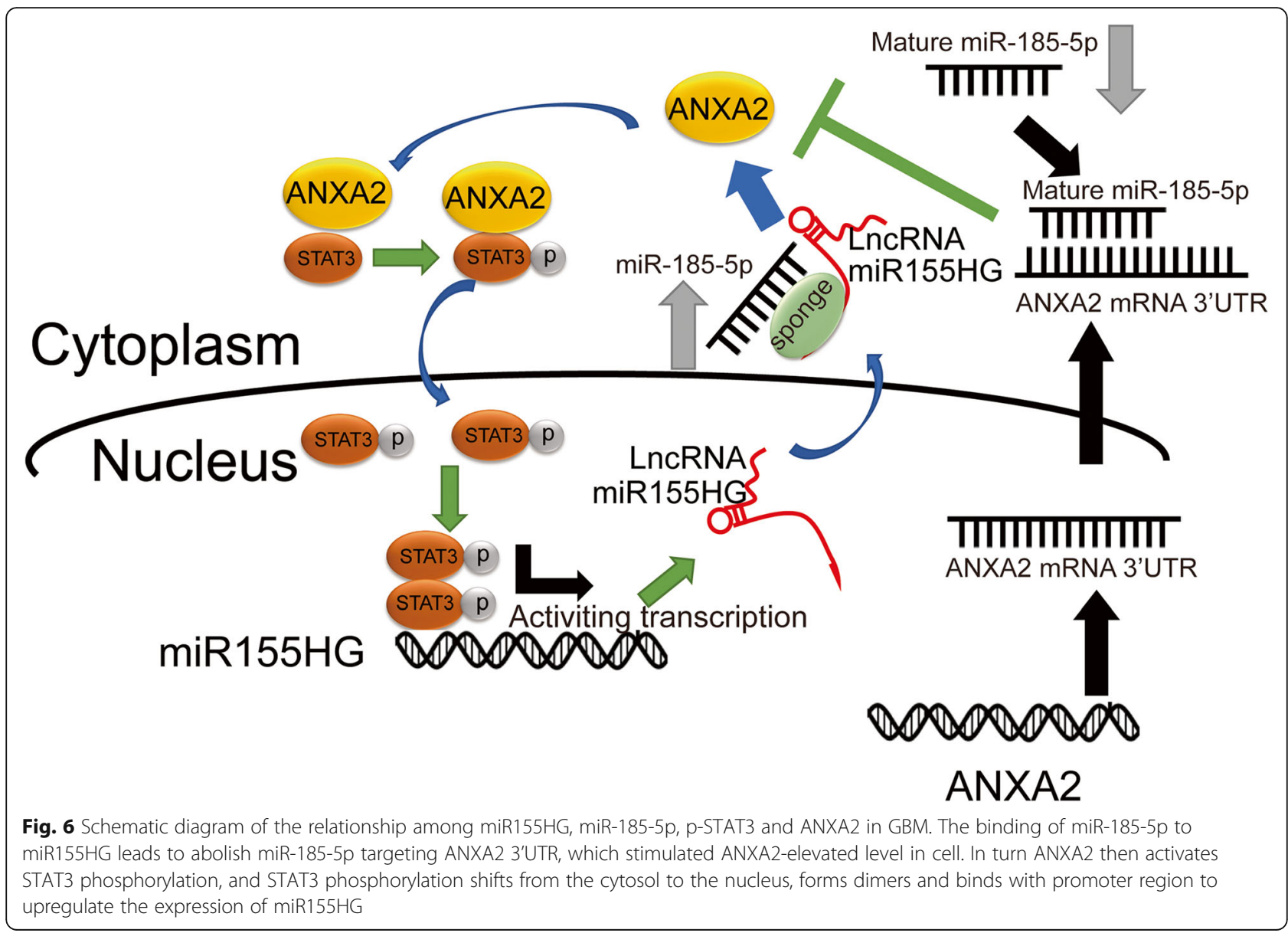

pancreatic cancer [48]. We found that ANXA2 was overexpressed in GBM. Based on TCGA, CGGA and Rembrandt GBM gene expression profiles, we found that ANXA2-associated genes were primarily enriched in cell proliferation and apoptosis. Knocking down ANXA2 inhibited proliferation and increased the G1/S cell cycle arrest apoptosis of U87 and GP1 cells. We also showed that ANXA2 is a key determining factor of survival that promotes the growth of intracranial GBM tumors in nude mice. We further found ANXA2 is a direct target of $\mathrm{miR}-185-5 \mathrm{p}$, and its expression was perturbed by miR-185-5p. Taken together, these results established the miR155HG/miR-185-5p/ANXA2 axis, which underlies the biological mechanisms of miR155HG in GBM.

Silencing ANXA2 was previously reported to inhibit activation of STAT3 (p-STAT3) [31, 32, 49], and we confirmed that ANXA2 knockdown decreased p-STAT3 in GBM cells. Activated p-STAT3 forms homologous dimers and enters into the nucleus to function as a transcription factor to promote target gene expression [50]. Since constitutively activated STAT3 is closely associated with GBM [51, 52], we find that ANXA2-mediated elevated miR155HG levels is mainly due to p-STAT3 level. ChIP assay and luciferase reporter gene assay showed that p-STAT3 could bind to the miR155HG promoter region from $-1548 \mathrm{bp}$ to $-1411 \mathrm{bp}$ upstream of the transcription start site to stimulate miR155HG expression. This suggests that STAT3 phosphorylation is the critical role in driving lncRNAs regulation similar to miR155HG in malignant brain tumor.

Here we have established miR155HG/miR-185-5p/ ANXA2 loop in GBM formation and progression. However, other interactions as epigenetic regulation between miR155HG and its downstream effector molecules remains obscure since lncRNA might recruit chromatin remodeling complex. More biological studies and clinical trials are needed to evaluate the practicality of targeting miR155HG for the treatment of GBM.

\section{Conclusions}

Our study suggested that the lncRNA miR155HG increases ANXA2 expression by sponging miR-185-5p to exert tumorigenic effects and that ANXA2 stimulates miR155HG level via ANXA2-driven p-STAT3 in GBM (Fig. 6). Thus, we have identified the miR155HG/ miR-185-5p/ANXA2 loop and its mechanisms and biological effects in malignant brain tumors. This loop could serve as a novel therapeutic biomarker for GBM. 


\section{Additional files}

Additional file 1: Figure S1. (A) U87 cells pretreated with a lentivirus with sh-miR155HG or sh-NC and a lentivirus containing luciferase were implanted in the brains of nude mice, and tumor formation was assessed by bioluminescence imaging. The bioluminescent images were measured at days 1,11 and 21 after implantation. (B) Overall survival from two groups of nude mice injected with U87 cells, transfected with sh-NC or sh-miR155HG lentivirus, was determined by Kaplan-Meier survival curves, and a log-rank test was used to assess the statistical significance of the differences. (C) Expression levels of ANXA2 in GBM tissues and adjacent normal brain tissues were analyzed by western blotting and normalized to $\beta$-catenin. (D) The protein expression levels of ANXA2 were analyzed by western blotting after $48 \mathrm{~h}$ transfection in U87 and GP1 cells with scramble, miR155HG siRNA 1, miR155HG siRNA 2, miR-NC or inhibitor, respectively. (E) The effect of sh-ANXA2 in U87 cell and tumor tissue of nude mice after implantation were analyzed by western blotting. (E) Downregulating ANXA2 contributed to the reduction of p-STAT3 level in GBM cells. (TIF $9482 \mathrm{~kb}$ )

Additional file 2: Figure S2. (A) Expression of ANXA2 in TCGA, CGGA and Rembrandt astrocytoma database. (B) ANXA2 associated genes from overlapping CGGA, TCGA and Rembrandt databases were analyzed with gene oncology analysis. (C) ANXA2 positively correlates with miR155HG in WHOII/III astrocytoma specimens of three independent public database. (TIF $3895 \mathrm{~kb}$ )

Additional file 3: Figure S3. (A) Expression levels of p-STAT3 in cell lines, GBM tissues and normal brain tissues were analyzed by western blot. (B) Downregulating ANXA2 contributed to the reduction of p-STAT3 level in GBM cells. (C) Overexpression STAT3 was constitutively activated by EGF in ANXA2depleted GBM cells in U87 and GP1 cells. (D) Luciferase assays was performed after transfection with miR155HG promoter wt-pGL3 or miR155HG promoer mut-pGL3 as well as the internal control Renilla plasmid into U87 and GP1 cells. The cells then were treated with or without SH-4-54. Relative luciferase activity was analyzed after $48 \mathrm{~h}$ treatment. $\left({ }^{*} p<0.05,{ }^{* *} p<0.01\right.$ ). (E) Two groups of representative immunohistochemical image of tumors from groups of nude mice implanted with U87 cells, transfected with a lentivirus with shmiR155HG or sh-NC, were shown to compare the volume size of tumors and the expression of ANXA2 and p-STAT3 through serial slices of same section of tumor. (TIF $13681 \mathrm{~kb}$ )

\section{Abbreviations}

EGF: Epidermal Growth Factor; CeRNAs: Competing endogenous RNAs; ChIP: Chromatin immunoprecipitation; FISH: Fluorescence in situ hybridization; GBM: Glioblastoma; GP1: Glioblastoma cell from patient 1; IHC: Immunoprecipitation; RIP: RNA immunoprecipitation

\section{Acknowledgments}

We thank Department of Neurosurgery, The First Affiliated Hospital of Nanjing Medical University (Nanjing, China) and Department of Neurosurgery, Nanjing First Hospital of Nanjing Medical University for their generous help. We thank Edanz Group (www.edanzediting.com/ac) for editing a draft of this manuscript.

\section{Funding}

This work was supported by grants from National Natural Science Foundation of China (81672501), Jiangsu Province's Science and Technology Foundation (BE2018724), Jiangsu Province's Natural Science Foundation (20170108), the Program for Advanced Talents within Six Industries of Jiangsu Province (2016-WSW-013), Jiangsu Province's Key Discipline of Medicine (ZDXKA2016001), and the Priority Academic Program Development of Jiangsu Higher Education Institutions (PAPD).

\section{Availability of data and materials}

The dataset(s) supporting the findings of this study are included within the article.

\section{Authors' contributions}

WWN, YTF and WYZ performed in vitro assays, collected clinical samples and analyzed the data; TW performed in vivo experiments; WWN wrote the manuscript; and WWN, ZJX and WYY designed this study. All authors have read and approved the final manuscript.

\section{Ethics approval and consent to participate}

The procedures of this study were approved by the Institutional Review Board of Nanjing Medical University.

\section{Consent for publication}

Not applicable.

\section{Competing interests}

The authors declare that they have no competing interests.

\section{Publisher's Note}

Springer Nature remains neutral with regard to jurisdictional claims in published maps and institutional affiliations.

\section{Author details}

${ }^{1}$ Department of Neurosurgery, The First Affiliated Hospital of Nanjing Medical University, Nanjing, Jiangsu 210029, China. ²Department of Neurosurgery, Nanjing First Hospital of Nanjing Medical University, Nanjing, Jiangsu, China.

Received: 14 November 2018 Accepted: 8 March 2019

Published online: 21 March 2019

\section{References}

1. Abou-Antoun TJ, Hale JS, Lathia JD, Dombrowski SM. Brain Cancer Stem Cells in Adults and Children: Cell Biology and Therapeutic ImplicationsNeurotherapeutics : the journal of the American Society for Experimental NeuroTherapeutics. 2017.

2. Patel AP, Tirosh I, Trombetta JJ, Shalek AK, Gillespie SM, Wakimoto H, et al. Single-cell RNA-seq highlights intratumoral heterogeneity in primary glioblastoma. Science (New York, NY). 2014;344:1396-401.

3. Bartonicek N, Maag $J$, Dinger ME. Long noncoding RNAs in cancer: mechanisms of action and technological advancements. Mol Cancer. 2016;15:43.

4. Kallen AN, Zhou XB, Xu J, Qiao C, Ma J, Yan L, et al. The imprinted H19 IncRNA antagonizes let-7 microRNAs. Mol Cell. 2013;52:101-12.

5. Elton TS, Selemon H, Elton SM, Parinandi NL. Regulation of the MIR155 host gene in physiological and pathological processes. Gene. 2013;532:1-12.

6. Haasch D, Chen YW, Reilly RM, Chiou XG, Koterski S, Smith ML, et al. T cell activation induces a noncoding RNA transcript sensitive to inhibition by immunosuppressant drugs and encoded by the proto-oncogene, BIC. Cell Immunol. 2002;217:78-86.

7. Kluiver J, Poppema S, de Jong D, Blokzijl T, Harms G, Jacobs S, et al. BIC and miR-155 are highly expressed in Hodgkin, primary mediastinal and diffuse large B cell lymphomas. J Pathol. 2005;207:243-9.

8. van den Berg A, Kroesen BJ, Kooistra K, de Jong D, Briggs J, Blokzijl T, et al. High expression of B-cell receptor inducible gene BIC in all subtypes of Hodgkin lymphoma. Genes, chromosomes \& cancer. 2003;37:20-8.

9. Neilsen PM, Noll JE, Mattiske S, Bracken CP, Gregory PA, Schulz RB, et al. Mutant p53 drives invasion in breast tumors through up-regulation of miR155. Oncogene. 2013;32:2992-3000.

10. Wu X, Wang Y, Yu T, Nie E, Hu Q, Wu W, et al. Blocking MIR155HG/miR-155 axis inhibits mesenchymal transition in glioma. Neuro-oncology. 2017;19: 1195-205.

11. Rescher U, Gerke V. Annexins--unique membrane binding proteins with diverse functions. J Cell Sci. 2004;117:2631-9.

12. Yang $T$, Peng $H$, Wang J, Yang J, Nice EC, Xie $K$, et al. Prognostic and diagnostic significance of annexin A2 in colorectal cancer. Colorectal disease : the official journal of the Association of Coloproctology of great. Britain and Ireland. 2013;15:e373-81.

13. Shetty PK, Thamake SI, Biswas S, Johansson SL, Vishwanatha JK. Reciprocal regulation of annexin A2 and EGFR with Her-2 in Her-2 negative and herceptin-resistant breast cancer. PLoS One. 2012;7:e44299.

14. Sharma MR, Koltowski L, Ownbey RT, Tuszynski GP, Sharma MC. Angiogenesis-associated protein annexin II in breast cancer: selective expression in invasive breast cancer and contribution to tumor invasion and progression. Exp Mol Pathol. 2006;81:146-56.

15. Takano S, Togawa A, Yoshitomi H, Shida T, Kimura F, Shimizu H, et al. Annexin II overexpression predicts rapid recurrence after surgery in 
pancreatic cancer patients undergoing gemcitabine-adjuvant chemotherapy. Ann Surg Oncol. 2008;15:3157-68.

16. Zhuang H, Tan M, Liu J, Hu Z, Liu D, Gao J, et al. Human epididymis protein 4 in association with Annexin II promotes invasion and metastasis of ovarian cancer cells. Mol Cancer. 2014;13:243.

17. Shetty P, Bargale A, Patil BR, Mohan R, Dinesh US, Vishwanatha JK, et al. Cell surface interaction of annexin $A 2$ and galectin-3 modulates epidermal growth factor receptor signaling in Her-2 negative breast cancer cells. Mol Cell Biochem. 2016;411:221-33.

18. Onishi M, Ichikawa T, Kurozumi K, Inoue $\mathrm{S}$, Maruo $\mathrm{T}$, Otani $\mathrm{Y}$, et al. Annexin A2 regulates angiogenesis and invasion phenotypes of malignant glioma. Brain tumor pathology. 2015;32:184-94.

19. Zhai H, Acharya S, Gravanis I, Mehmood S, Seidman RJ, Shroyer KR, et al. Annexin A2 promotes glioma cell invasion and tumor progression. J Neurosci. 2011:31:14346-60.

20. Maruo T, Ichikawa T, Kanzaki H, Inoue S, Kurozumi K, Onishi M, et al. Proteomics-based analysis of invasion-related proteins in malignant gliomas. Neuropathology : official journal of the Japanese Society of Neuropathology. 2013;33:264-75.

21. Wu W, Zhou X, Yu T, Bao Z, Zhi T, Jiang $K$, et al. The malignancy of miR-18a in human glioblastoma via directly targeting CBX7. Am J Cancer Res. 2017;7:64-76.

22. Wu W, Hu Q, Nie E, Yu T, Wu Y, Zhi T, et al. Hypoxia induces H19 expression through direct and indirect Hif-1alpha activity, promoting oncogenic effects in glioblastoma. Sci Rep. 2017;7:45029.

23. Tian C, Deng Y, Jin Y, Shi S, Bi H. Long non-coding RNA RNCR3 promotes prostate cancer progression through targeting miR-185-5p. Am J Transl Res. 2018;10:1562-70.

24. Afshar S, Najafi R, Sedighi Pashaki A, Sharifi M, Nikzad S, Gholami MH, et al. MiR-185 enhances radiosensitivity of colorectal cancer cells by targeting IGF1R and IGF2. Biomedicine \& pharmacotherapy = Biomedecine \& pharmacotherapie. 2018;106:763-9.

25. Zhang Q, Chen Y, Liu K. miR-185 inhibits cell migration and invasion of hepatocellular carcinoma through CDC42. Oncol Lett. 2018;16:3101-7.

26. Zhao L, Zhang Y, Liu J, Yin W, Jin D, Wang D, et al. MiR-185 inhibits cell proliferation and invasion of non-small cell lung cancer by targeting KLF7. Oncol Res. 2018.

27. Filipowicz W, Bhattacharyya SN, Sonenberg N. Mechanisms of posttranscriptional regulation by microRNAs: are the answers in sight? Nat Rev Genet. 2008:9:102-14.

28. Schumperli D, Pillai RS. The special Sm core structure of the U7 snRNP: farreaching significance of a small nuclear ribonucleoprotein. Cellular and molecular life sciences : CMLS. 2004:61:2560-70.

29. Su SC, Maxwell SA, Bayless KJ. Annexin 2 regulates endothelial morphogenesis by controlling AKT activation and junctional integrity. J Biol Chem. 2010;285:40624-34.

30. Zhang F, Wang Z, Yuan J, Wei X, Tian R, Niu R. RNAi-mediated silencing of Anxa2 inhibits breast cancer cell proliferation by downregulating cyclin D1 in STAT3-dependent pathway. Breast Cancer Res Treat. 2015;153:263-75.

31. Maji S, Chaudhary P, Akopova I, Nguyen PM, Hare RJ, Gryczynski I, et al. Exosomal Annexin II promotes angiogenesis and breast Cancer metastasis. Molecular cancer research : MCR. 2017;15:93-105.

32. Wang T, Yuan J, Zhang J, Tian R, Ji W, Zhou Y et al. Anxa2 binds to STAT3 and promotes epithelial to mesenchymal transition in breast cancer cells. Oncotarget. 2015;6:30975-92

33. Lo HW, Hsu SC, Xia W, Cao X, Shih JY, Wei Y, et al. Epidermal growth factor receptor cooperates with signal transducer and activator of transcription 3 to induce epithelial-mesenchymal transition in cancer cells via up-regulation of TWIST gene expression. Cancer Res. 2007:67:9066-76.

34. Davis FM, Azimi I, Faville RA, Peters AA, Jalink K, Putney JW Jr, et al. Induction of epithelial-mesenchymal transition (EMT) in breast cancer cells is calcium signal dependent. Oncogene. 2014;33:2307-16.

35. Ebert MS, Sharp PA. Emerging roles for natural microRNA sponges. Curr Biol. 2010;20:R858-61.

36. Li S, Ma Y, Hou X, Liu Y, Li K, Xu S, et al. MiR-185 acts as a tumor suppressor by targeting AKT1 in non-small cell lung cancer cells. Int J Clin Exp Pathol. 2015;8:11854-62

37. Li Q, Wang JX, He YQ, Feng C, Zhang XJ, Sheng JQ, et al. MicroRNA-185 regulates chemotherapeutic sensitivity in gastric cancer by targeting apoptosis repressor with caspase recruitment domain. Cell Death Dis. 2014; 5:e1197.
38. Qadir XV, Han C, Lu D, Zhang J, Wu T. miR-185 inhibits hepatocellular carcinoma growth by targeting the DNMT1/PTEN/Akt pathway. Am J Pathol. 2014;184:2355-64.

39. Liu C, Chen Z, Hu X, Wang L, Li C, Xue J, et al. MicroRNA-185 downregulates androgen receptor expression in the LNCaP prostate carcinoma cell line. Mol Med Rep. 2015;11:4625-32.

40. Tang H, Wang Z, Liu X, Liu Q, Xu G, Li G, et al. LRRC4 inhibits glioma cell growth and invasion through a miR-185-dependent pathway. Curr Cancer Drug Targets. 2012;12:1032-42.

41. Tran DDH, Kessler C, Niehus SE, Mahnkopf M, Koch A, Tamura T. Myc target gene, long intergenic noncoding RNA, Linc00176 in hepatocellular carcinoma regulates cell cycle and cell survival by titrating tumor suppressor microRNAs. Oncogene. 2018;37:75-85.

42. Boyko V, Mudrak O, Svetlova M, Negishi Y, Ariga H, Tomilin N. A major cellular substrate for protein kinases, annexin II, is a DNA-binding protein. FEBS Lett. 1994;345:139-42.

43. Krutilina RI, Babich VS, Kropotov AV, Mudrak OS, Chesnokov IN, Turoverova $L V$, et al. The DNA-binding activity of the membrane protein annexin II and its interaction with antibodies to the chromatin nuclear ribonucleoprotein (alpha-RNP). Tsitologiia. 1996;38:1106-14.

44. Bao H, Jiang M, Zhu M, Sheng F, Ruan J, Ruan C. Overexpression of Annexin II affects the proliferation, apoptosis, invasion and production of proangiogenic factors in multiple myeloma. Int J Hematol. 2009;90:177-85.

45. Lokman NA. Ween MP, Oehler MK, Ricciardelli C. The role of annexin A2 in tumorigenesis and cancer progression. Cancer microenvironment : official journal of the International Cancer Microenvironment Society. 2011;4:199-208.

46. Kohli S, Bhardwaj A, Kumari R, Das S. SIRT6 is a target of regulation by UBE3A that contributes to liver tumorigenesis in an ANXA2-dependent manner. Cancer Res. 2018;78:645-58.

47. Rocha MR, Barcellos-de-Souza P, Sousa-Squiavinato ACM, Fernandes PV, de Oliveira IM, Boroni M. Annexin A2 overexpression associates with colorectal cancer invasiveness and TGF-ss induced epithelial mesenchymal transition via SrC/ANXA2/STAT3. 2018:8:11285

48. Keklikoglou I, Hosaka K, Bender C, Bott A, Koerner C, Mitra D, et al. MicroRNA-206 functions as a pleiotropic modulator of cell proliferation, invasion and lymphangiogenesis in pancreatic adenocarcinoma by targeting ANXA2 and KRAS genes. Oncogene. 2015:34:4867-78.

49. Yuan J, Yang Y, Gao Z, Wang Z, Ji W, Song W, et al. Tyr23 phosphorylation of Anxa2 enhances STAT3 activation and promotes proliferation and invasion of breast cancer cells. Breast Cancer Res Treat. 2017;164:327-40.

50. Aaronson DS, Horvath CM. A road map for those who don't know JAKSTAT, vol. 296. New York, NY: Science; 2002. p. 1653-5.

51. Villalva C, Martin-Lanneree S, Cortes U, Dkhissi F, Wager M, Le Corf A, et al. STAT3 is essential for the maintenance of neurosphere-initiating tumor cells in patients with glioblastomas: a potential for targeted therapy? Int J Cancer. 2011:128:826-38

52. McFarland BC, Gray GK, Nozell SE, Hong SW, Benveniste EN. Activation of the NF-kappaB pathway by the STAT3 inhibitor JSI-124 in human glioblastoma cells. Mol Cancer Res. 2013;11:494-505.

Ready to submit your research? Choose BMC and benefit from:

- fast, convenient online submission

- thorough peer review by experienced researchers in your field

- rapid publication on acceptance

- support for research data, including large and complex data types

- gold Open Access which fosters wider collaboration and increased citations

- maximum visibility for your research: over $100 \mathrm{M}$ website views per year

At $\mathrm{BMC}$, research is always in progress.

Learn more biomedcentral.com/submission 